\title{
Comportement d'éprouvettes de mortiers et de bétons à la mer
}

\author{
Mohamed Boutouil \\ Doctorant, LMFGC, Université du Houre
}

Daniel Caminade

Maitre de conférences, $L M F G C$, Université du Havre

Daniel Levacher

Professeur des Universités, LMFGC, Université du Hovre

Franck Schoefs

Doctorant, agrégé, LMG, Université de Nantes

\section{Résumé}

L'étude de la durabilité des bétons soumis à l'action du milieu marin, a fait l'objet en 1974 d'un programme de recherche spécifique

Cet article présente les résultats obtenus pour 14 ciments à différentes teneurs en $\mathrm{C}_{3} \mathrm{~A}$ et $\mathrm{SO}_{3}$, sur le comportement à long terme :

- des mortiers : essais de traction et de compression à 15 ans.

- des bétons : mesure de la vitesse du son à 20 ans.

Les résultats confirment les bonnes qualités des ciments avec ajout de laitiers (ex. CPAL et CLK).

\section{1- Introduction}

Pour concevoir des ouvrages en béton, béton armé ou béton précontraint destinés à être utilisés dans un milieu agressif tel que le milieu marin, on doit s'assurer au préalable que le matériau de base (le béton), possède des qualités durables pour la vie des ouvrages.

La durabilité du béton peut être appréciée comme sa capacité à conserver ses caractéristiques dimensionnelles et mécaniques (résistance, élasticité) qui permettront d'éviter les désordres (fissures, éclatements, épaufrures...) qui, en facilitant la pénétration des constituants de l'eau de mer, pourraient entrainer la ruine de l'ouvrage par destruction du béton ou par corrosion des armatures et des câbles de précontrainte.

La durabilité du béton en milieu marin dépend des conditions d'exposition, de la constitution du béton (compacité, granularité, dosage en ciment) et de la qualité de ces constituants, principalement le ciment.

En France, la Commission Permanente des Liants hydrauliques et Adjuvants du Béton, COPLA a homologué une catégorie de ciment destinée aux structures exposées à l'action de la mer ou des embruns : le ciment prise mer. 
Ces ciments sont caractérisés par de faibles teneurs en aluminate tricalcique $\left(\mathrm{C}_{3} \mathrm{~A}<10 \%\right)$ et en sulfates $\left(\mathrm{SO}_{3}<2.5 \%\right)$.

Afin d'étudier le comportement de divers ciments et de mesurer l'influence des teneurs en $\mathrm{C}_{3} \mathrm{~A}$ et $\mathrm{SO}_{3}$ sur la durabilité du béton en milieu marin, la COPLA a entrepris en 1974 un programme d'essais sur une longue durée : vingt ans.

\section{2- Programme d'essais (COPLA)}

Le programme initial de la COPLA était d'étudier l'évolution de mortiers et de bétons constitués à partir de ciments (voir tableau 1) sélectionnés sur trois sites: Le Havre, La Rochelle et Marseille. Actuellement, seul le site du Havre continue les essais.

\begin{tabular}{|c|c|c|c|c|c|c|c|c|}
\hline \multirow[t]{2}{*}{ Type de ciment } & \multicolumn{2}{|c|}{ Teneur en } & \multirow{2}{*}{$\begin{array}{l}\text { Année de } \\
\text { fabrication }\end{array}$} & \multicolumn{5}{|c|}{ bétons } \\
\hline & $\mathrm{C}_{3} \mathrm{~A} \%$ & $\mathrm{SO}_{3} \%$ & & $\mathrm{C}(\mathrm{kg})$ & $\mathrm{G}(\mathrm{kg})$ & $\mathrm{S}(\mathrm{kg})$ & $E(1)$ & $\mathrm{E} / \mathrm{C}$ \\
\hline $1:$ CPA 400 & $<5$ & $\# 2.5$ & 1974 & 350 & 1160 & 719 & 160 & 0.457 \\
\hline 1 Bis : CPA 325 & $<5$ & $\# 2.5$ & 1974 & 350 & 1160 & 702 & 160 & 0.457 \\
\hline 2: CPA 325 & $\# 8$ & $\# 2.5$ & 1974 & 350 & 1160 & 697 & 168 & 0.48 \\
\hline 3: CPA 325 & $\# 8$ & $\# 3.5$ & 1974 & 350 & 1160 & 684 & 173 & 0.494 \\
\hline 4: CPA 400 & $\# 8$ & $\# 3.5$ & 1974 & 350 & 1160 & 695 & 168 & 0.48 \\
\hline $5:$ CPA 400 & $\# 8$ & $\# 4.5$ & 1974 & 350 & 1160 & 690 & 172 & 0.491 \\
\hline $6:$ CPA 400 & $\# 14$ & $\# 3.5$ & 1974 & 350 & 1160 & 685 & 172 & 0.491 \\
\hline $7:$ CPA 325 & $\# 10$ & $\# 2.5$ & 1974 & 350 & 1160 & 684 & 175 & 0.5 \\
\hline $\begin{array}{l}8: \text { CPAL } 325 \\
20 \% \text { laitiers }\end{array}$ & $>14$ & NC & 1974 & 350 & 1160 & 690 & 170 & 0.485 \\
\hline $\begin{array}{l}9: \text { CPF } 325 \\
30 \% \text { laitiers }\end{array}$ & $>14$ & $\mathrm{NC}$ & 1974 & 350 & 1160 & 676 & 175 & 0.5 \\
\hline $\begin{array}{l}10: \text { CPF } 325 \\
30 \% \text { laitiers }\end{array}$ & $\# 10$ & $\mathrm{NC}$ & 1974 & 350 & 1160 & 695 & 165 & 0.471 \\
\hline $\begin{array}{r}11: \text { CLK } 325 \\
80 \% \text { laitiers } \\
\end{array}$ & $\begin{array}{r}\text { à faib } \\
\text { en } \mathrm{CaO}\end{array}$ & $\begin{array}{l}\text { teneur } \\
48.57 \% \text { ) }\end{array}$ & 1974 & 350 & 1160 & 680 & 172 & 0.491 \\
\hline 12 : Cim Prompt & $\mathrm{NC}$ & $\mathrm{NC}$ & 1976 & 450 & 1061 & 572 & 215 & 0.477 \\
\hline $13: \mathrm{CPAZ} 400$ & $\mathrm{NC}$ & $\mathrm{NC}$ & 1978 & 350 & 1140 & 700 & 175 & 0.5 \\
\hline
\end{tabular}

$\mathrm{NC}$ : non connu

Tableau 1 : liste des ciments retenus pour les essais.

\section{2-1 Eprouvettes de mortier}

Réalisées en mortier normalisé et conservées en eau de mer (après 28 jours), elles font l'objet d'essais de traction par flexion et d'essais de compression $(7 \mathrm{j}, 28 \mathrm{j}$, $90 \mathrm{j}, 1$ an, 2 ans, 5 ans, 10 ans, 15 ans et 20 ans)

\section{2-2 Eprouvettes de béton}

Les éprouvettes prismatiques $\left(20^{*} 20 * 80 \mathrm{~cm}\right)$ et cylindriques $(16 * 32 \mathrm{~cm})$ ont été confectionnées avec des bétons dosés à $350 \mathrm{~kg}$ de ciment et comportant $1160 \mathrm{~kg}$ de graviers. Le dosage en sable et en eau a été ajusté en vue d'obtenir une ouvrabilité identique (\#6 cm d'affaissement au cône d'Abrams). 
Outre les 12 ciments testés initialement (éprouvettes confectionnées en 1974), deux autres catégories ont été ajoutées en 1976 et 1978 avec des compositions de bétons légèrement différentes.

Le tableau 1 donne les indications essentielles sur la nature des ciments testés et sur la composition des bétons correspondants.

Sur toutes les éprouvettes de béton, on mesure la vitesse du son par auscultation dynamique ( $28 \mathrm{j}, 90 \mathrm{j}, 1$ an, 2 ans, 5 ans, 10 ans, 15 ans et 20 ans) grâce à un appareil prototype mis au point par le CETE de Rouen.

Il est prévu à l'échéance de 20 ans de procéder à des essais de traction par flexion et de compression sur les prismes $20 * 20 * 80 \mathrm{~cm}$.

Les essais mécaniques sur les cylindres $16^{*} 32 \mathrm{~cm}$ sont répertoriés sur le tableau 2 .

\begin{tabular}{|c|c|c|}
\hline $\begin{array}{c}\text { Type d'essais } \rightarrow \\
\text { Mode de conservation } \downarrow\end{array}$ & Traction par fendage & Compression \\
\hline Eau douce & 28 jours et 20 ans & 28 jours et 20 ans \\
\hline Embruns & 2 ans & 2 ans \\
\hline Zone de marnage & 2 ans, 10 ans et 20 ans & 2 aiss, 10 ans et 20 ans \\
\hline
\end{tabular}

Tableau 2 : Essais mécaniques et leurs échéances.

\section{2-3 Analyse des résultats}

Les résultats présentés ici portent sur l'analyse des essais réalisés sur :

1 - les mortiers (essais de traction et compression à 15 ans )

2- les bétons (mesures des vitesses du son à 20 ans)

Les comparaisons sont faites pour chacun des ciments en prenant pour base les valeurs à 90 jours qui semblent plus adéquates pour juger des évolutions à long terme que la valeur traditionnelle à 28 jours.

\section{3-Evolution des résistances des mortiers}

La comparaison est effectuée sur une période de 15 ans. En outre il faut noter que les éprouvettes correspondant aux ciments 6 et 8 à fortes teneurs en $C_{3} A$, conservées en milieu marin ont été détruites en moins de 2 ans et ne sont pas analysées ici. Sur les figures 1 et 2 , les catégories non représentées ont des rapports de résistances $(\mathrm{R} / \mathrm{R} 90 \mathrm{j})$ très peu différents de 1 .

\section{3-1 Evoldtion des résistances moyennes en compression (figure 1)}

A part le ciment $n^{\circ} 1$ (CPA 325) qui accuse une perte allant jusqu'à $20 \%$ à 15 ans, on constate une stabilisation et une amélioration des caractéristiques, à long terme, des mortiers. Le gain de résistance concerne surtout des ciments contenant une proportion notable de laitier, ce qui semble cohérent avec ce que l'on sait de l'évolution de ce type de ciment. Parmi les ciments CPA, les catégories 4 et 5 semblent être affectées, à moyen terme ( 5 et 10 ans), par le mode de conservation en eau de mer. Les teneurs élevées en $\mathrm{SO}_{3}$ (supérieures à $3 \%: 3.4$ et $4.46 \%$ respectivement) seraient à l'origine de ce comportement. 


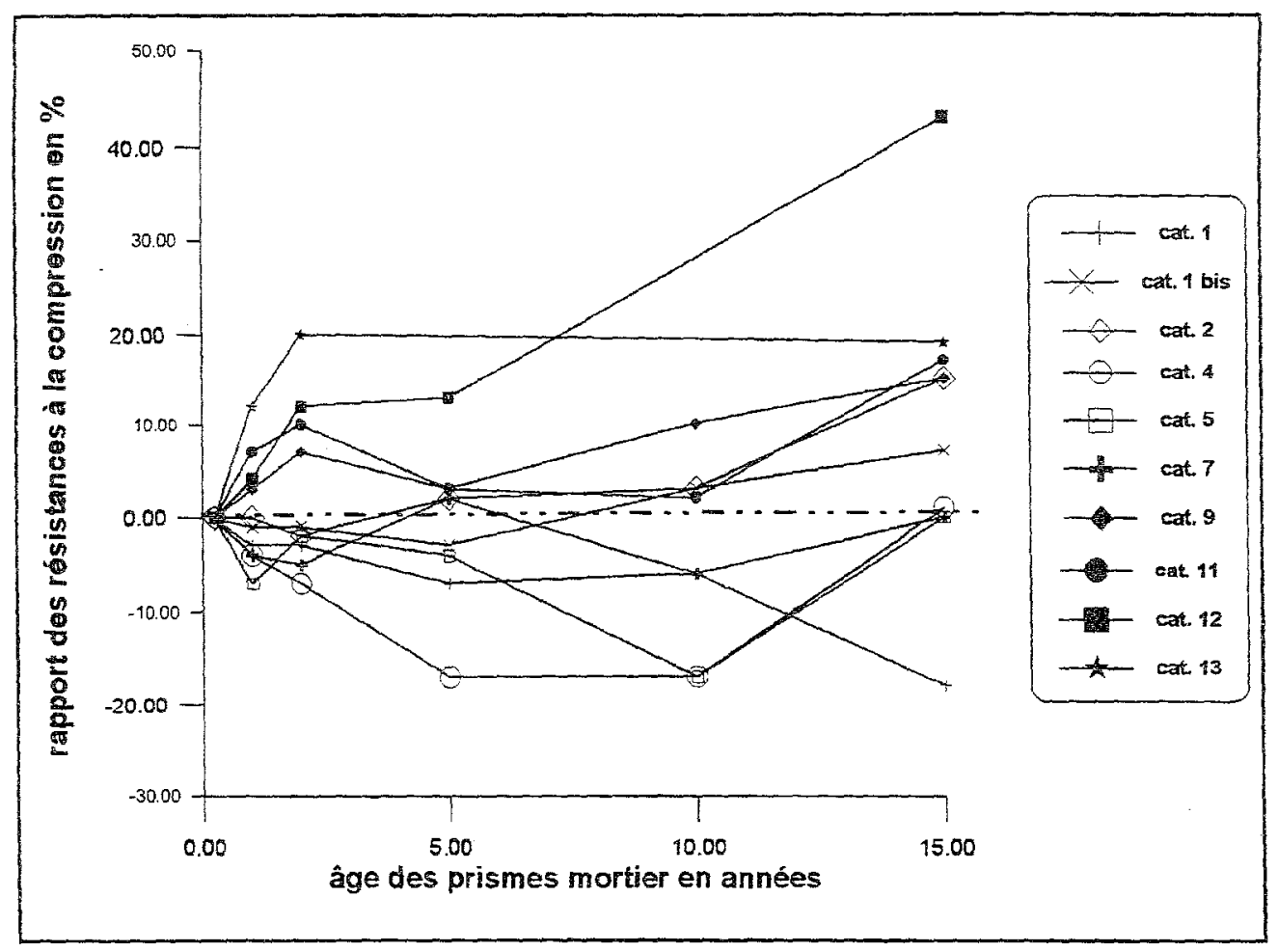

Figure 1: Evolution du rapport des résistances à la compression ( R / R $90 \mathrm{j})$

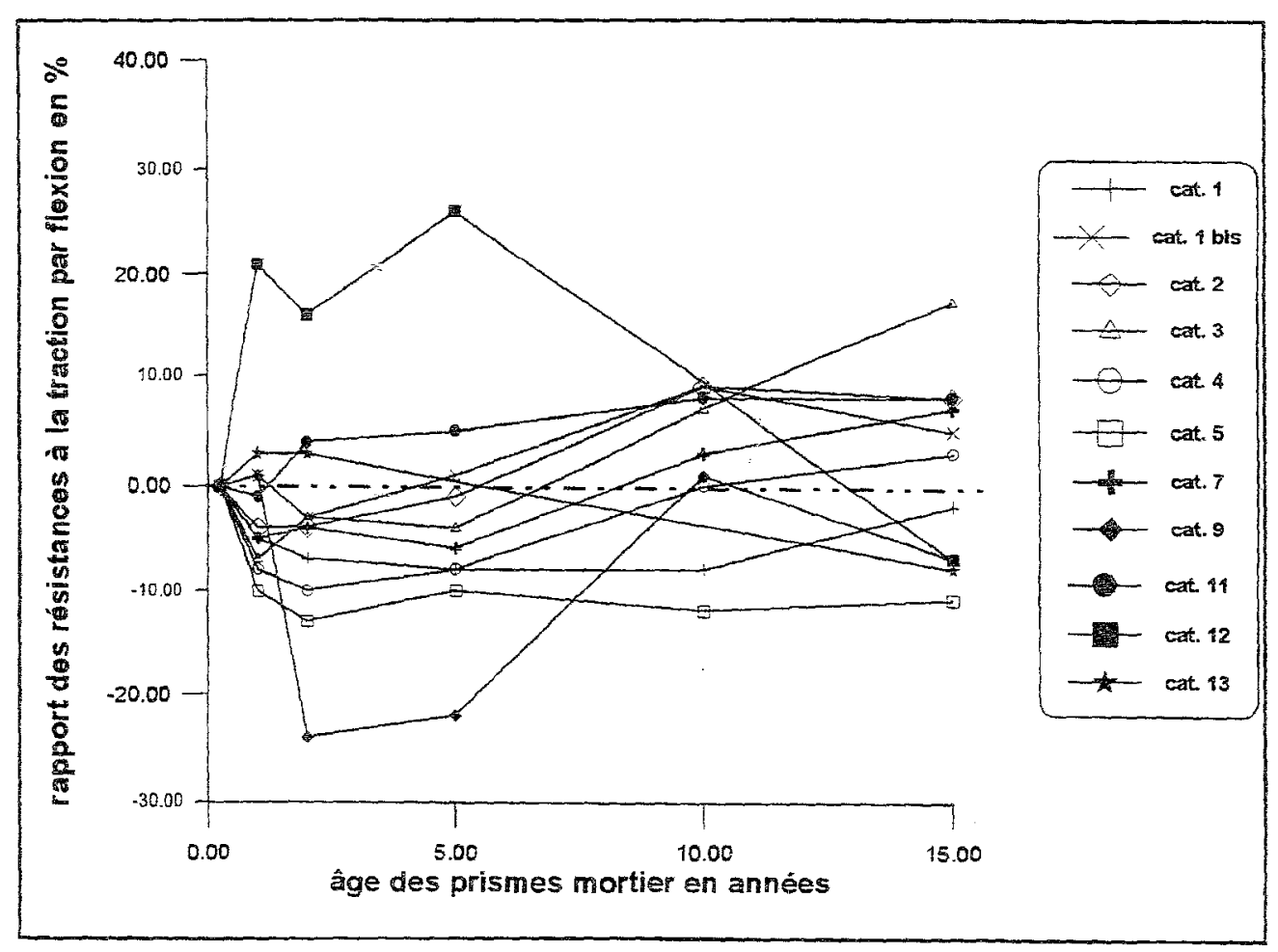

Figure 2: Evolution du rapport des résistances à la traction par flexion (R/R $90 j)$ 


\section{3-2 Evolution des résistances moyennes à la traction par flexion (figure 2)}

Les résultats obtenus sont ici beaucoup plus dispersés et il semblerait que les ciments CPA évoluent, en général, plus favorablement que les ciments aux laitiers. Par ailleurs, pour la majorité des ciments, on constate que les résistances à la flexion semblent être légèrement plus affectées par l'eau de mer que les résistances à la compression. Cette dégradation est visible dès $90 \mathrm{j}$. Toutefois, à partir de 2 ans, on observe une tendance au confortement que l'on peut expliquer par l'hypothèse de la formation d'une couche de surface qui constituerait une protection pour les éprouvettes. La couche de surface est constituée d'aragonite/calcite $\left(\mathrm{CaCO}_{3}\right)$ et/ou de brucite $\left(\mathrm{Mg}(\mathrm{OH})_{2}\right)$ et mesure moins d'un $\mathrm{mm}$ d'épaisseur après 20 ans d'exposition à l'eau de mer.

En s'intéressant au cas de la catégorie 5 , on constate que la dégradation observée en compression est confirmée par une perte de résistance à la traction. Cette catégorie possède la teneur la plus élevée en $\mathrm{SO}_{3}: 4.46 \%$.

Parmi les ciments aux laitiers, la catégorie 11 présente un comportement caractérisé par un gain en résistance à la traction et confirme la bonne tenue à l'eau de mer du ce ciment à $80 \%$ de laitiers.

\section{3-3 Analyse de la dispersion des résultats}

Compte tenu de la dispersion habituellement observée pour ce type d'essais et tout particulièrement pour les essais de traction par flexion, nous avons réalisé une analyse de cette dispersion par l'intermédiaire du coefficient de variation C.O.V. 1 est défini par le rapport de l'ecart-type $\sigma$ à la moyenne $\mathrm{m}: \mathrm{C} . \mathrm{O} \cdot \mathrm{V}=\sigma / \mathrm{m}$.

Pour les essais de traction, ce coefficient est compris entre 5 et $10 \%$ et, est un peu pius faible, que pour les essais en compression où il varie de 5 à $15 \%$. Les valeurs atteintes indiquent une dispersion raisonnable des valeurs mesurées, eu égard au type d'essai réalisé.

\section{4- Evolution des yitesses de transmission du son}

La mesure des vitesses du son a été réalisée grâce à un appareil prototype mis au point par le CETE de Rouen. Les mesures effectuées permettent de suivre globalement, et sans détruire les éprouvettes, l'évolution des bétons et en particulier de mettre en évidence l'apparition de modifications internes de la structure telles que des fissures ou des micro-fissures qui entrainent une chute notable de la vitesse de propagation du son.

On compare ici l'évolution du comportement des bétons (éprouvettes cylindriques $16^{*} 32 \mathrm{~cm}$ ), conservés en eau douce et en zone de marnage, sur une période de 20 ans. 


\section{4-1 Eprouvettes conservées en ean douce (figure 3)}

Pour la plupart des éprouvettes, on constate une augmentation modérée de la vitesse du son. Cette augmentation est particulièrement visible entre $90 \mathrm{j}$ et 2 ans. Elle correspondrait au durcissement du béton qui s'avérera par la suite influencé par la conservation en zone de marnage.

Après 2 ans, les variations de la vitesse du son sont assez légères et ne dépassent guère ies $5 \%$.

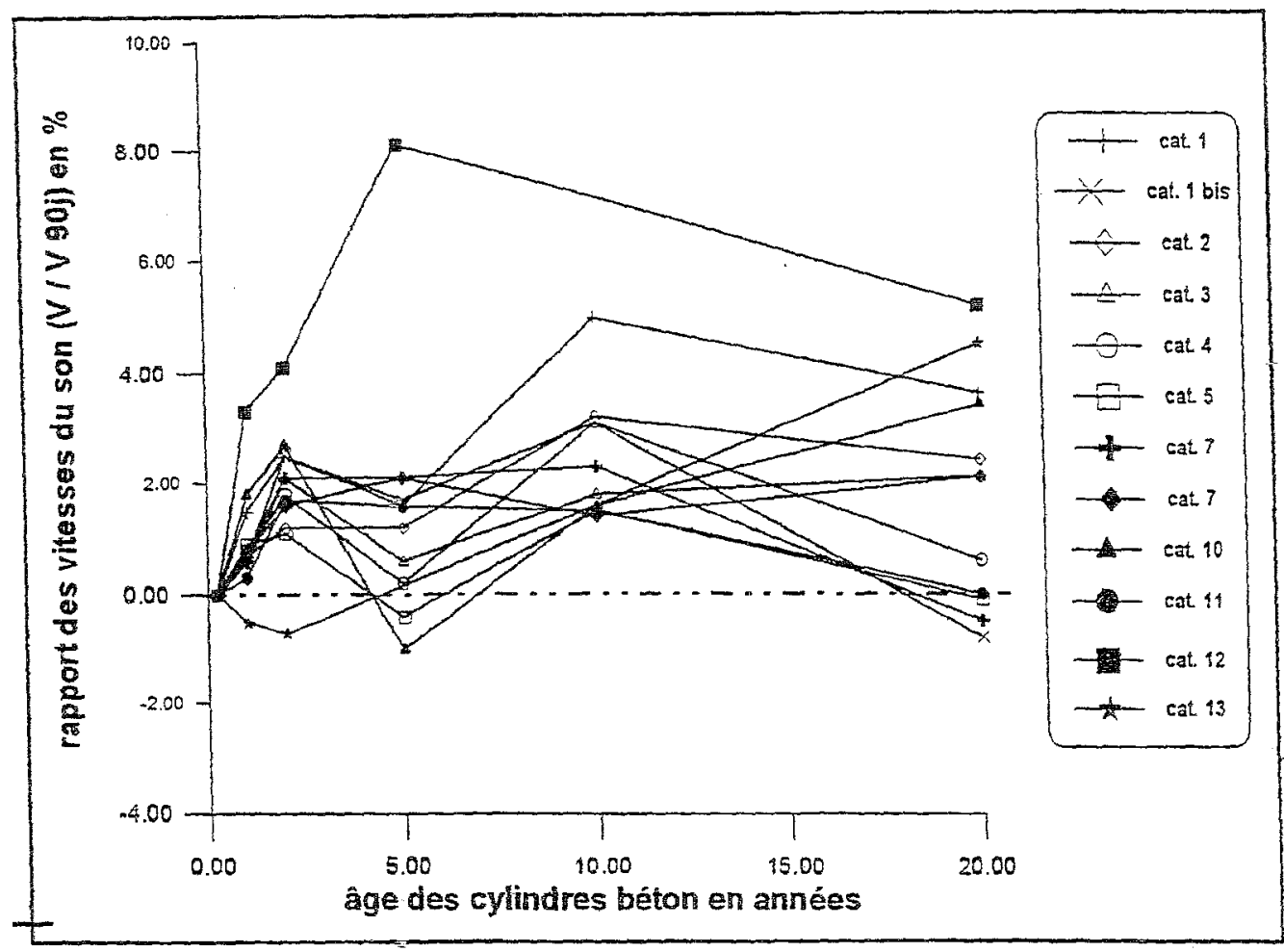

Figure 3 : Evolution du rapport des vitesses du son (éprouvettes en eau douce)

\section{4-2 Eprouvettes conservées en zone de marnage (figure 4)}

Les ciments de type CPA subissent en général, une chute de la vitesse de transmission du son, alors que les ciments aux laitiers voient cette vitesse augmenter. Il semblerait que ces ciments supportent mieux l'action du milieu marin.

En outre, l'évolution de la vitesse du son fait apparaître une moindre augmentation de celle-ci entre $90 j$ et 2 ans que dans le cas de la conservation en eau douce, montrant ainsi que le durcissement semble être influencé par la conservation en zone de marnage. 


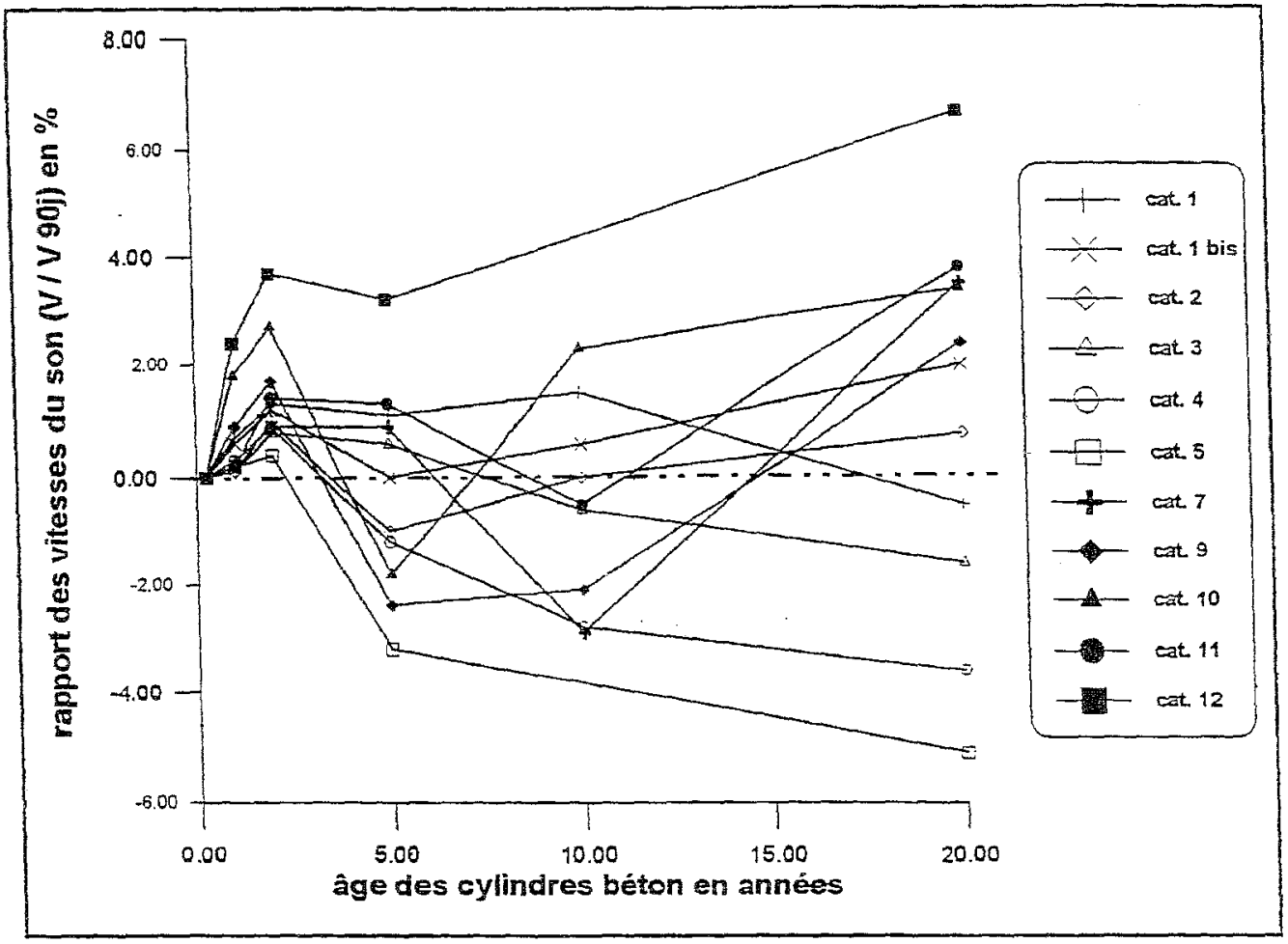

Figure 4: Evolution du rapport des vitesses du son (éprouvettes en zone de marnage)

\section{5-Conclusion (tableau 3 )}

Le suivi du comportement en eau de mer des quatorze ciments a mis en évidence des résultats prévisibles sur la sensibilité des ciments aux attaques chimiques suivant les teneurs en $\mathrm{C}_{3} \mathrm{~A}$ et $\mathrm{SO}_{3}$ (voir tableau 3). En effet, les éprouvettes en ciment à teneur très élevée en $C_{3} A$ (\#14\%) ont été complètement détruites après deux ans (ciments 6 et 8 ). Une teneur en $\mathrm{SO}_{3}$ supérieure à $3 \%$ a engendré une mauvaise une tenue à l'eau de mer (ciments 4 et 5). Par rapport aux valeurs obtenues à $90 \mathrm{j}$, on a constaté une diminution des caractéristiques mécaniques des mortiers (résistances à la compression et à la traction par flexion) et de la vitesse de transmission du son dans les bétons. Les ciments avec ajouts de laitiers de hauts fourneaux ont montré une bonne résistance à l'action agressive de l' eau de mer.

Les essais à 20 ans qui comportent des essais complets de compression et de traction, y compris les prismes $20 * 20 * 80 \mathrm{~cm}$, devraient permettre une appréciation plus précise de l'évolution des caractéristiques à long terme des bétons soumis à l'action du milieu marin. 


\begin{tabular}{|c|c|c|c|c|c|c|c|c|c|c|c|c|c|c|}
\hline $\begin{array}{l}\text { ciments } \rightarrow \\
\text { types d'essais } \downarrow\end{array}$ & 1 & $\begin{array}{c}1 \\
\text { bis }\end{array}$ & 2 & 3 & 4 & 5 & 6 & 7 & 8 & 9 & 10 & 11 & 12 & 13 \\
\hline $\begin{array}{l}\text { Mortiers (15 ans) } \\
\text { - Compression } \\
\text { - Traction }\end{array}$ & $=$ & $\begin{array}{l}+ \\
+\end{array}$ & $\begin{array}{l}++ \\
+\end{array}$ & $\begin{array}{l}= \\
++\end{array}$ & $\begin{array}{l}= \\
+\end{array}$ & $\begin{array}{l}= \\
--\end{array}$ & $\begin{array}{l}D \\
E \\
T\end{array}$ & $\begin{array}{l}= \\
+\end{array}$ & $\begin{array}{l}\mathrm{D} \\
\mathrm{E} \\
\mathrm{T}\end{array}$ & + & $\begin{array}{l}= \\
=\end{array}$ & $\begin{array}{l}+ \\
+ \\
\end{array}$ & $\begin{array}{l}+1 \\
- \\
\end{array}$ & $\begin{array}{c}++ \\
- \\
\end{array}$ \\
\hline $\begin{array}{l}\text { Bétons : vitesse du } \\
\text { son (20 ans) } \\
\text { - Eau douce } \\
\text { - Mamage }\end{array}$ & $\begin{array}{l}++ \\
=\end{array}$ & - & $\begin{array}{l}++ \\
=\end{array}$ & + & $\begin{array}{l}= \\
-\end{array}$ & $=$ & $\begin{array}{l}\mathrm{R} \\
\mathrm{U} \\
\mathrm{I} \\
\mathrm{T}\end{array}$ & $\begin{array}{l}= \\
+\end{array}$ & $\begin{array}{l}R \\
U \\
I \\
T\end{array}$ & $\begin{array}{l}+ \\
+ \\
\end{array}$ & $\begin{array}{l}+ \\
+\end{array}$ & $\begin{array}{l}= \\
+\end{array}$ & $\begin{array}{l}++ \\
+\end{array}$ & $\stackrel{+}{\mathrm{NC}}$ \\
\hline
\end{tabular}

$+:$ forte augmentation, $>10 \%$ pour les mortiers, $>5 \%$ pour les bétons

$+:$ augmentation, entre 2 et $10 \%$ pour les mortiers, 1 et $5 \%$ pour les bétons

$=:$ faible variation en plus ou moins, entre 0 et $2 \%$ pour les mortiers, 0 et $1 \%$ pour les betons

$-:$ diminution

-- : forte diminution

Tableau 3: Variation de la grandeur mesurée à 15 ans (résistances) ou à 20 ans (vitesse du son) par rapport à celle à $90 \mathrm{j}$

\section{Références bibliographiques}

AM. PAILLERE, M. RAVERDY, J.MULLET, «Influence du ciment sur la dégradation du béton en milieu marin ». Bull. Liaison LCPC 135, jan-fév 1985, pp. 5-10

A.M. PAILLERE, M. RAVERDY, «Etude de longue durée, de l'influence de la composition minéralogique des ciments sur la tenue à l'eau de mer. Essai dans l'eau de mer artificielle et dans la Manche $\gg .3^{\circ}$ conférence CANMET/ACI, Nice, mai 1994

M. BOUTOUIL, «Comportement d'éprouvettes de mortiers et de bétons à la mer». Mémoire de DEA, Université de Nantes (LMG), Université du Havre (LMFGC), 1995, $85 \mathrm{p}$

P. POTTEVIN, "Durability of plain and reinforced concrete in marine structureFrench practice $\gg . \mathrm{K}$. and B. mather international conference/ACI Atlanta, 1987, pp. 281-303 